Théologiques

Théologiques

\title{
Orientation lacanienne sur les rites
}

\section{Guy-Robert St-Arnaud}

Volume 4, numéro 2, octobre 1996

L'épreuve du rien

URI : https://id.erudit.org/iderudit/602443ar

DOI : https://doi.org/10.7202/602443ar

Aller au sommaire du numéro

\section{Éditeur(s)}

Faculté de théologie de l'Université de Montréal

\section{ISSN}

1188-7109 (imprimé)

1492-1413 (numérique)

Découvrir la revue

\section{Citer cet article}

St-Arnaud, G.-R. (1996). Orientation lacanienne sur les rites. Théologiques, 4(2), 117-131. https://doi.org/10.7202/602443ar

\section{Résumé de l'article}

Rites et religion ne conduisent pas inexorablement au constat freudien de la névrose universelle. En effet, les pratiques rituelles n'occupent pas un seul discours. Cette diversité ne soustrait pas les rites au point de vue freudien mais conduit à une autre lecture, celle de l'opération des signifiants. L'orientation lacanienne permet de la mettre en évidence et de la déployer dans sa façon particulière de produire des effets de sens. d'utilisation que vous pouvez consulter en ligne.

https://apropos.erudit.org/fr/usagers/politique-dutilisation/ 


\title{
Orientation lacanienne sur les rites ${ }^{1}$
}

\author{
Guy-Robert ST-ARNAUD \\ Faculté de théologie \\ Université de Montréal
}

\section{RÉSUMÉ}

Rites et religion ne conduisent pas inexorablement au constat freudien de la névrose universelle. En effet, les pratiques rituelles n'occupent pas un seul discours. Cette diversité ne soustrait pas les rites au point de vue freudien mais conduit à une autre lecture, celle de l'opération des signifiants. L'orientation lacanienne permet de la mettre en évidence et de la déployer dans sa façon particulière de produire des effers de sens.

Rites and religion do not lead inexorably to the Freudian claim of a universal neurosis. In effect, ritual practices do not occupy a single discourse. This diversity does not remove rites from a Freudian point of view but leads to another reading, that of the operation of signifiers. A Lacanian orientation reveals and displays this operation in its particular fashion of producing effects of meaning.

Les rites revêtent un aspect symbolique. Freud reconnaît sans conteste cette caractéristique au rite à caractère religieux et au cérémonial névrotique (STARNAUD, 1996). Or, une telle dimension symbolique laisse cours à des lectures différentes. S'inscrivant sous l'égide d'un retour à Freud, la perspective psychanalytique de Jacques Lacan permet-elle d'affiner une lecture freudienne des rites?

Ce texte est constitué de quatre parties. La première présente brièvement quelques rapports entre les problématiques freudienne et lacanienne. $\mathrm{La}$ deuxième précise une place particulière que Lacan attribue aux rites. Or, ce rôle dévolu aux rites les associe à une forme de réitération mise à jour dans une

1 Ce texte constitue une suite autonome de l'article "Aspect freudien des rites et de leurs répétitions" se trouvant dans le numéro intitulé "Les Rites : céder en résistant " de Théologiques, vol. 4/1, mars 1996. 
troisième partie. Finalement, le développement lacanien conduit à des conséquences fournissant un éclairage sur la dynamique des rites.

\section{Aperçu des rapports entre les problématiques freudienne et lacanienne}

Un bilan succinct de la perspective freudienne sur les rites révèle au moins trois résultats. Dans un premier temps, le lien entre les rites et la réduplication de protection, constitutive de la névrose obsessionnelle, s'avère intrinsèque. Cependant, ce lien n'est pas exclusif à l'obsession pour autant. Aussi, les foules donnent lieu à d'autres modalités de récurrence faisant surgir des formes rituelles associées davantage à l'hystérie qu'à l'obsession. Un deuxième résultat met en évidence le double renversement opéré par l'élaboration de Freud. Alors que les actes obsédants étaient relayés à une absence de sens, la découverte de l'inconscient freudien instaure l'avènement de ceux-ci au champ de la cohérence et du sens. De ce fait, la distinction fondée sur le sens des rites religieux par opposition au non-sens du cérémonial névrotique devient obsolète.

Le troisième résultat établit un parallélisme entre les motifs de l'acte obsédant et ceux du cérémonial religieux. Si les raisons déterminantes du cérémonial névrotique sont inconnues au névrosé, Freud écrit : "Les mobiles qui poussent impérieusement les croyants aux exercices religieux leur restent cependant à tous inconnus, ou bien sont représentés dans leur conscience par d'autres mobiles mis en avant à leur place " (FREUD, 1989, p. 89). Ce non-savoir à propos du sens convie les rites à une aporie. D'une part, ignorer le sens du rite laisse place à l'illusion et à la névrose. D'autre part, préciser le sens éveille la résistance puisqu'il mène à l'angoisse sous-jacente ayant donné lieu à l'accomplissement et à l'élaboration du rite lui-même.

Ce dilemme plonge donc les rites dans une impasse ${ }^{2}$. Il ne s'agit pas seulement d'un beau risque où la volonté seule suffirait à pallier à l'indifférence ou à la crainte de s'engager dans un rite. Son accomplissement ne s'avère pas irrémédiablement couronné de la signification susceptible de résorber l'angoisse. En somme, l'aporie posée par la problématique freudienne n'est pas anodine et l'esquive devient malaisée. Aussi, la problématique lacanienne ne va pas à l'encontre des acquis freudiens et tente d'en dégager leur structure. $A$ la suite de Freud, trois éléments attirent l'attention.

Un premier élément a trait à la dimension universelle. La pratique psychanalytique a conduit Freud à lire des traces de névrose chez les personnes enclines à des pratiques rituelles. Aussi, préférer l'option consistant à ignorer les

2 Voir ST-ARNAUD, 1996, p. 90-91. Rappelons que cette impasse ne dépend pas du sens symbolique ou non des rites. Selon Freud, les actes obsédants ont aussi une représentation symbolique. 
mobiles d'une telle tendance peut, certes, éviter le développement d'une névrose individuelle comme le mentionne Freud : « ..., la religion réussit à épargner à quantité d'êtres humains une névrose individuelle * (FREUD, 1981, p. 31). Cet évitement semble bien ténu puisque Freud considère la religion comme une forme symptomatique généralisée : « la religion serait la névrose obsessionnelle universelle de l'humanité;... " (FREUD, 1989, p. 61; nous soulignons). Ce verdict freudien n'annonce pas la fin imminente de la religion et de ses formes rituelles multiples!

Sur ce point, Lacan ne reprend pas la perspective de Freud avec les mêmes termes. Il aborde la religion avec les trois catégories du réel, du symbolique et de l'imaginaire. Le symbolique correspond à la dimension langagière et aux signifiants, l'imaginaire à la réalité qui soutient la représentation, et le réel à ce qui est inévitable. Selon la formulation lacanienne, la religion réalise le symbolique de l'imaginaire. En somme, la dimension religieuse fait constamment retour en évoquant le mot dernier sur la réalité (voir LACAN, 1966, p. 872), avec la part d'illusion qui s'y rattache. À la suite de Freud, Lacan considère la religion en tant qu'elle perdure :

Eh bien, ce qui réalise le Symbolique de l'imaginaire, qu'est-ce que c'est d'autre que la religion? pour moi! Ce qui réalise, en termes propres, le symbolique de l'imaginaire, c'est bien ce qui fait que la religion n'est pas près de finir. (LACAN, 1973-74, 13 novembre 1973; nous soulignons)

Freud indique une généralisation de la forme symptomatique à plusieurs personnes tandis que Lacan souligne une autre forme d'universalité, à savoir une généralisation dans le temps. Pendant que des sociologues et des philosophes des années 70 annonçaient la fin imminente de la religion, Lacan marquait la dynamique de sa continuité. Le constat contemporain établi par Danièle Hervieu-Léger semble corroborer le précédent propos lacanien de 1973 :

Il y a vingt-cinq ans, lorsqu'on entreprenait de s'initier à la sociologie religieuse, les choses étaient relativement simples. Il s'agissait, pour l'essentiel, d'entrer dans l'analyse de la perte contemporaine de la religion. Cette éviction de la religion était alors considérée comme l'un des traits majeurs de la modernité. [...]

Vingt ans plus tard, le paysage de la recherche apparaît étonnamment modifié, ... La religion, qu'on disait refoulée a la marge des sociétés avancées, ne serait-elle pas en train de démontrer sa capacité de retrouver une nouvelle pertinence sociale, politique et culturelle, dans une modernité en crise? (HERVIEU-LÉGER, 1994, p. 31-32; nous soulignons)

Si la modernité escompte faire fi de la religion et de ses manifestations, l'aporie freudienne et la perspective lacanienne convergent sur la question de leur place. Ce premier élément concernant la dimension universelle peut 
paraître paradoxal. D'un côté, Freud et Lacan soulignent l'universalité de la religion et de ses rites. De l'autre, les rites présentent une entrée suspecte et constituent une impasse. Pourquoi des formes multiples de rites se développentelles ou connaissent-elles une expansion universelle alors que leur existence se révèle si problématique d'un point de vue psychanalytique? Le point suivant fournit une indication à ce sujet.

Un deuxième élément soulevé par la perspective freudienne concerne le lien entre les rites et l'angoisse. La tendance à s'adonner à des pratiques rituelles surgit sur une toile de fond souvent teintée d'angoisse. Les rites constituent alors une forme de réponse à cette angoisse. Ils deviennent des indicateurs de la présence de l'angoisse. De ce fait, les rites sont des effets de celle-ci et peuvent se réduire à un pis-aller masquant les causes. En ce sens, les rites entretiennent l'illusion et développent la névrose. Freud a très bien cerné cette difficulté lorsqu'il mentionne que les mobiles des rites sont souvent inconnus ou cachés par d'autres motifs secondaires. Donc, les rites présentent un certain attrait mais deviennent alors un cadeau empoisonné, un baume méphitique. Chez Freud, les pratiques rituelles se trouvent ainsi entachées d'un aspect névrotique. La problématique lacanienne ne présente pas d'opposition à ce point de vue. Toutefois, elle recèle une approche montrant leur apport et leur place singulière faisant l'objet de ce qui suit.

Le troisième élément ouvre une nouvelle orientation. La perspective freudienne annihile la distinction entre rite religieux et cérémonial névrotique. En effet, les deux pratiques rituelles se trouvent assujetties à du sens qui opère à l'insu du croyant comme du névrosé. De la considération de l'inconscient, Freud pose un lieu où un savoir est supposé, indépendamment de la conscience que les sujets peuvent en avoir.

Champs religieux et psychanalytique se retrouvent au lieu commun d'une supposition de savoir. La problématique freudienne du paradoxe des rites, religieux ou autres, établit un lieu où le savoir sur les mobiles et le sens des rites peut être supposé. Selon Lacan, la supposition de savoir pour un sujet trouve une articulation particulière :

Dès qu'il y a quelque part le sujet supposé savoir - que je vous ai abrégé aujourd'hui au haut du tableau par S. s. S. - il y a transfert. (LACAN, 1963. 64, p. 210)

Ces considérations orientent donc la problématique de la place des rites vers une approche lacanienne du transfert. Lacan ne présente pas d'études spécifiques sur les rites religieux comparés aux cérémonials névrotiques. La mise en évidence d'un lieu porteur de la supposition de savoir permet de poursuivre la lecture au point précis où Freud achève son investigation. Telle est la pertinence de la perspective lacanienne sur le transfert. 


\section{Transfert et place du rite selon Lacan}

En psychanalyse, le transfert est constitué de la relation entre un analysant ${ }^{3}$ et un analyste. Celle-ci débute avec les demandes de l'analysant évoquant les raisons d'entreprendre une analyse. Ces demandes mettent en jeu un sujet supposé savoir ce qu'il en est de la cause des souffrances de l'analysant. Au début de l'analyse, la place où se situe cette supposition de savoir s'appelle analyste.

Regrouper rites religieux et cérémonials névrotiques sous cet espace commun d'une supposition de savoir conduit à un retournement. Cette fois, c'est le champ religieux qui interroge la psychanalyse. Ce lieu d'une supposition de savoir ne favorise-t-il pas des expressions rituelles dans la pratique même de l'analyse?

En effet, il n'est pas rare que des formes de rituels s'installent au cœur même du défilement des séances d'analyse, aussi bien de la part de l'analyste que de l'analysant. Gestes itératifs où le manteau ou la main tendue de la salutation se cabre sous le même pli, mouvements de tâtonnements où l'analysant allongé cherche à retrouver son moule corporel avant de commencer à dire, toutes ces manifestations constituent autant d'éléments qui se répètent avec la minutie du rite. Comment la place d'analyste peut-elle prendre acte d'un développement rituel à l'intérieur même de la pratique analytique, ou d'un analysant qui parle en séance d'un rite auquel il a participé?

Orienter ce parcours vers la question du transfert ne confine pas le rite au champ du religieux, le plaçant sous la lorgnette suspicieuse de la psychanalyse. Au contraire, le transfert et les répétitions sous diverses formes touchent la psychanalyse au sein de sa pratique. Les champs religieux et psychanalytique sont tous les deux concernés par la place des rites et la façon dont opère la répétition.

Depuis les débuts de la psychanalyse, le concept de transfert se trouve associé à la problématique de la répétition. L'article de Freud intitulé Remémoration, répétition et perlaboration, daté de 1914, pose un dilemme entre remémoration et répétition. L'analysant traduit en actes dans la relation de transfert quelque chose dont il n'a aucune idée. Par exemple, il ne se souvient pas et ne dit pas la crainte éprouvée face à un parent alors qu'il agit ainsi à l'égard de l'analyste. L'analysant répète plutôt que de se remémorer, et en plus « le malade répète évidemment cet acte sans savoir qu'il s'agit d'une répétition »(FREUD, 1989,

3

La perspective lacanienne instaure cette orthographe, et non pas celle de psychanalysé ou analysé. Cet aspect souligne la responsabilité du dit de la personne qui effectue un travail analytique. De plus, il est question de la place de l'analyste et non plus seulement de la personne du psychanalyste. Cette distinction indique d'emblée qu'une analyse n'a pas à conduire à une identification à la personnalité du psychanalyste (voir LACAN, 1953-54, p. 129; 1956-57, p. 26). 
p. 108). Puisque l'analysant va jusqu'à ignorer qu'il est en train de répéter, la répétition implique cette place de l'analyste où le savoir peut être supposé. En ce sens, il s'agit d'un double appel au transfert. La répétition se rattache directement au transfert parce que la relation entre analysant et analyste sert à mettre en scène l'oubli ou l'indicible, et aussi parce que la répétition convoque le lieu d'analyste comme supposé savoir que ça répète :

C'est ici que la présence du psychanalyste est irréductible, comme témoin de cette perte. (LACAN, 1963-64, p. 116)

Le transfert constitue un temps de fermeture du discours de l'inconscient. La description imagée de ce mouvement de fermeture faite par Lacan dresse le bilan de cette entrée au lieu de l'inconscient :

Mais les choses sont moins faciles, parce que c'est une entrée où l'on n'arrive jamais qu'au moment où l'on ferme (cette place ne sera jamais touristique), et que le seul moyen pour qu'elle s'entrouvre, c'est d'appeler de l'intérieur. (LACAN, 1966, p. 838)

Ce temps de fermeture de l'inconscient, Freud le lit comme un manque à la remémoration au profit de la répétition se manifestant dans le transfert. Lorsqu'il traite de la répétition associée à l'inconscient, Lacan fait mention du rite à propos de l'interprétation d'un rêve rapporté par Freud (1980, p. 433). Un père a veillé jour et nuit son enfant malade. Après la mort de l'enfant, un vieillard est chargé de la veillée mortuaire près du cercueil entouré de cierges. Pendant ce temps, le père se repose dans une chambre à côté et il fait le rêve suivant : "l'enfant est près de son lit, lui prend le bras, et murmure d'un ton plein de reproche : "Ne vois-tu donc pas que je brûle?" ". Le père s'éveille et trouve un bras du petit cadavre brûlé par un cierge qui a tombé tandis que le veilleur s'était assoupi.

Selon Freud, le rêve est un accomplissement de désir. Alors qu'intervient la satisfaction de prolonger le sommeil comme venant seconder le désir inconscient, Lacan interroge la place du désir sous l'aspect du réel comme rencontre manquée. L'intérêt du rêve réside dans la mise en place du désir par la voie de ce qui échappe cruellement à une rencontre père-fils. La perte cruelle de l'objet fait de cette rencontre une épreuve :

Car ce n'est pas que, dans le rêve, il se soutienne que le fils vit encore. Mais l'enfant mort prenant son père par le bras, vision atroce, désigne un au-dela qui se fait entendre dans le rêve. Le désir s'y présentifie de la perte imagée au point le plus cruel de l'objet. C'est dans le rêve seulement que peut se faire cette rencontre vraiment unique.(LACAN, 1963-64, p. 58; nous soulignons)

Lacan situe le rite dans une perspective de répétition. Il n'est autre que l'unique répondant à l'unicité de la place du rêve. De ce rêve où s'accomplit du désir inconscient commémorant l'absence de remémoration, le rite en devient 
l'exclusif pendant diurne : "Seul un rite, un acte toujours répété, peut commémorer cette rencontre immémorable ... » (LACAN, 1963-64, p. 58; nous soulignons). Si le rêve est accomplissement de désir, le rite est-il écriture ${ }^{4}$ de l'impossible accomplissement d'un désir qui n'a de cesse? Se répétant, le rite trace le suspens du désir qui ne cesse pas de ne pas s'écrire 5 .

Alors que le rêve porte le suspens du désir pendant le sommeil, le rite constitue la voie royale de l'inconscient par sa façon de mettre en jeu la répétition. La suite de ce parcours précise comment la répétition se relie au signifiant.

\section{La répétition signifiante ${ }^{6}$ et les rites}

Aborder le rite comme prise en charge du réel d'une rencontre manquée convie à toucher de plus près l'articulation entre inconscient et répétition. Dans une première étape, il s'agit d'établir un rapport entre l'inconscient et le langage. Lacan établit ce lien par le biais du concept de signifiant que la seconde étape précise. Le signifiant permet au sujet de surgir. Cet avènement du sujet est marqué d'une division et d'une perte. La troisième étape établit un rapprochement entre le réel de cette perte et l'instauration d'une dynamique de répétition. Finalement, les rites et l'opération du signifiant se trouvent conjoints en une même forme de répétition.

\subsection{Inconscient et langage}

Selon Lacan, l'inconscient est structuré comme un langage. Cette perspective, il la retrouve chez Freud. Le travail du rêve s'effectue selon la condensation et le déplacement que Lacan met en correspondance avec la métaphore et la métonymie (FREUD, 1980, p. 241-266; LACAN, 1966, p. 493-523). En référence à la linguistique (A. KREMER-MARIETTI, 1978, p. 105-190; A. LEMAIRE, 1977, p. 69-98, 135-235), Lacan décrit ces deux figures de style par des relations entre signifiants.

Dans la métonymie, la relation entre les signifiants suspend l'effet de sens, c'est-à-dire instaure un déplacement du signifié. Par exemple, "Il a bu un verre." constitue une métonymie qui reporte le signifié du contenu et du

4 Cette perspective n'est pas développée dans cet article dont la demande portait sur un point de chute orienté directement à partir du champ psychanalytique. Voir R. SUBLON, 1993, p. 123-176 où est abordée la question des sacrements.

5 Lacan utilise cette formulation pour présenter la modalité de l'impossible.

6 La répétition comporte aussi une dimension pulsionnelle qui a une incidence sur les rites. Ces deux modalités de répétition, pulsionnelle et signifiante, relèvent d'un processus discursif particulier associé au concept lacanien de Lettre. Seul un aperçu de la répétition signifiante est présenté dans cet atticle. 
nombre de verres sur le contenant. L'exemple précédent peut être utilisé pour dire de façon voilée qu'une personne a bu plusieurs verres de boisson.

L'effet de sens produit par la métaphore manifeste une condensation où les signifiants se trouvent dans une relation de surimposition. L'émergence de la signification de l'expression, "le soleil de mon cœur... ", marque une chaleureuse intensité dépassant ce qui tient à cœur.

Ni la métaphore ni la métonymie ne se réfèrent à un signifiant isolé qui porterait à lui seul tout le sens. Dans les deux figures de style, l'effet de sens surgit du rapport entre les signifiants.

\subsection{Signifiant et surgissement d'un sujet divisé}

Pour Lacan, le signifiant ne se signifie pas lui-même (LACAN, 1955-56, p. 207-231). Il opère intrinsèquement de la différence :

Nous nous servons du signifiant - qui n'est pas un signifiant pour lui, mais qui, pour fonctionner comme signifiant, doit s'inscrire dans une différence ... (LACAN, 1963-64, p. 207)

Aussi, cette dynamique de la différence par rapport aux autres signifiants définit le signifiant comme étant ce qui représente le sujet pour un autre signifiant :

L'effet de langage, c'est la cause introduite dans le sujet. ... Car sa cause, c'est le signifiant sans lequel il n'y aurait aucun sujet dans le réel. Mais ce sujet, c'est ce que le signifiant représente, et il ne saurait rien représenter que pour un autre signifiant... (LACAN, 1966, p. 835)

Non seulement le signifiant ne représente pas lui-même mais le sujet ne surgit dans le réel que de l'effet d'entre-deux et de report d'un signifiant à l'autre : "le sujet est littéralement, à son origine, et comme tel, l'élision d'un signifiant, le signifiant sauté dans la chaîne. "(LACAN, 1959-60, p. 264) Un signifiant représentant entièrement le sujet s'avère manquant. L'avènement du sujet au cœur du " manque-au-signifié " d'un signifiant instaure une dynamique de réitération. Lacan mentionne l'importance de cet aspect pour comprendre l'intérêt que l'humain porte au retour des cycles naturels et aux rites associés :

..., vous y verrez l'explication de bien des choses, ne serait-ce que cette singularité repérable dans l'histoire qui s'appelle les rites. Je veux dire ces rites par quoi l'homme des civilisations dites primitives se croit obligé d'accompagner la chose la plus naturelle du monde, à savoir le retour des cycles précisément naturels. [...]

Quel rapport peut-il y avoir entre l'homme et le retour du lever du soleil? si ce n'est que, comme homme parlant, il se sustente dans un rapport direct au signifiant. (LACAN, 1959-60, p. 264) 
Sujet du langage, l'être parlé n'est parlant qu'assujetti. L'inscription au champ du langage a un effet " d'a-sujet " puisque son avènement de sujet réside dans sa perte au lieu de l'Autre. Le lieu de l'Autre, défini comme lieu du trésor du signifiant (LACAN, 1966, p. 806), divise le sujet entre l'enfermement sous un signifiant, réduit à ne rien signifier lui-même, et les vagues d'une représentation qui se défile au gré du ballottement de signifiant en signifiant. Dès lors qu'il parle, l'être humain se trouve marqué d'une perte, soit une inscription dans un ordre signifiant. Les signifiants parlent le sujet, le causent et l'entretiennent.

La division du sujet parlant par les signifiants provoque un reste qui ne se résorbe pas, un manque-à-être. Ce manque est constitutif du sujet pris dans cet écart entre les signifiants, entre le dire et le dit, entre l'être et le sens. Inéluctable, ce manque-à-être ne cesse de faire retour et forme une présentification du réel : "Le réel est ici ce qui revient toujours à la même place... " (LACAN, 1963-64, p. 49; 1973-74, 23 avril 1974). Le manque fait retour dans la chaîne des signifiants.

Pour Lacan, réel et réalité sont distincts. Alors que la réalité laisse la possibilité d'ignorer les objets de notre environnement, le réel lacanien est impossible à éviter même s'il s'avère insaisissable : " un rendez-vous auquel nous sommes toujours appelés avec un réel qui se dérobe " (LACAN, 1963-64, p. 53; nous soulignons). Lacan l'écrit objet $a$, objet petit a.

\subsection{Réel et dynamique de répétition}

Le réel est de l'ordre de "l'a-rencontre " impossible, de la tuché. Le rêve du père éveillé par les murmures de la voix de son enfant mort, "Ne vois-tu donc pas que je brûle? ", fait écho à cette rencontre manquée : "Mais l'enfant mort prenant son père par le bras, vision atroce, désigne un au-delà qui se fait entendre dans le rêve. " (LACAN, 1963-64, p. 58; nous soulignons) Même si cette situation est empreinte d'un ratage, les signifiants du rêveur résonnent pourtant avec insistance.

Les signifiants faisant entendre cette vision atroce illustre ce rapport entre la tuché et l'automaton. Si la tuché marque la rencontre manquée, l'automaton correspond au réseau de signifiants :

[...] le rapport qu'Aristote établit entre l'automaton - et nous savons, au point où nous en sommes de la mathématique moderne, que c'est le réseau de signifiants - et ce qu'il désigne comme la tuché - qui est pour nous la rencontre du réel. (LACAN, 1963-64, p. 51; nous soulignons)

Structuré comme un langage, l'inconscient est déterminé par cet automaton, le réseau des signifiants : " [...] au sens précis où nous enseignons que l'inconscient, c'est que l'homme soit habité par le signifiant,... " (LACAN, 
1966, p. 35). Lacan situe le principe de la répétition dans l'insistance du réseau de signifiants constituant une chaîne signifiante :

Notre recherche nous a mené à ce point de reconnaître que l'automatisme de répétition (Wiederholungszwang) prend son principe dans ce que nous avons appelé l'insistance de la chaîne signifiante. (LACAN, 1966, p. 11)

La chaîne signifiante ne se constitue pas du simple défilement des mots. Elle répète avec une telle intensité que Freud et Lacan y relèvent un aspect de contrainte :

Et le Zwang [du Wiederholungszwang], la contrainte, que Freud définit par la Wiederholung [répétition], commande les détours mêmes du processus primaire [processus de l'inconscient]. (LACAN, 1963-64, p. 55, nous soulignons)

La contrainte est telle que la chaîne signifiante constitue un automatisme qui répète, soit l'automaton. Le réel, l'objet $a$, forme une sorte de point de butée du fait qu'il échappe constamment à la chaîne signifiante? ${ }^{7}$. Le réel n'est pas la répétition en tant que telle. Cependant, chaque nouvelle reprise de la chaîne signifiante défaille au même lieu impossible. Inévitable point d'achoppement, la tuché se retrouve en permanence à l'affût de l'automatisme de répétition :

Nous l'avons [à propos du terme tuché emprunté à Aristote] traduit par la rencontre du réel. Le réel est au-delà de l'automaton, du retour, de la revenue, de l'insistance des signes à quoi nous voyons commandés par le principe du plaisir. Le réel est cela qui gît toujours derriere l'automaton, ... (LACAN, 1963-64, p. 54; nous soulignons)

Le réel étant situé hors de la chaîne signifiante, il ne s'y trouve toujours que par défaut, c'est-à-dire manquant. En contrepartie, l'insistance de la chaîne signifiante conduit à la répétition.

\subsection{Automatisme de répétition et rites}

Le retour au même lieu de la tuché distingue l'automatisme de répétition de la reproduction. Lacan retrace cette différence dans l'élaboration freudienne à partir du moment où Freud délaisse la catharsis orientée essentiellement vers le souvenir originel :

7 Le réel agit comme un hors-signifié dont Lacan fournit une certaine idée à partir de l'instinct de mort : « C'est très certainement ce que Freud nous a apporté sous le terme d'instinct de mort. Il s'agit de cette limite du signifié qui n'est jamais atteinte par aucun être vivant, ou même, qui n'est jamais atteinte du tout, ...) (LACAN, 1956-57, p. 48). 
[...], je vous indique que, dans les textes de Freud, répétition n'est pas reproduction. Jamais d'oscillation sur ce point - Wiederholen n'est pas Reproduzieren.

Reproduire, c'est ce qu'on croyait pouvoir faire au temps des grands espoirs de la catharsis. On avait la scène primitive en reproduction comme on a aujourd'hui les tableaux de maîtres pour neuf francs cinquante. Seulement, ce que Freud nous indique quand il fait ses pas suivants, et il ne met pas longtemps à les faire, c'est que rien ne peut être saisi, ni détruit, ni brûlé, sinon de façon, comme on dit, symbolique, in effigie, in absentia.

La répétition apparaît d'abord sous une forme qui n'est pas claire, qui ne va pas de soi, comme une reproduction, ou une présentification, en acte. (LACAN, 1963-64, p. 49-50)

La différence entre répétition et reproduction relève de l'acte. La catharsis vise à retrouver le souvenir de ce qui fut au début. Aussi, la reproduction tient à la similarité des éléments de départ et d'arrivée. En revanche, la répétition manifeste que le même se trouve au cœur de l'accomplissement d'un acte (dire, agir, écrire, lire,...). Les deux séquences suivantes de caractère fournissent une idée de la disparité de ces deux perspectives :

Séquence \#1:

\section{Séquence $\# 2$ :}

\section{reproduction}

répétition

La première séquence illustre la reproduction. La différence entre chacun des caractères repose sur la modulation, la variation. L'un change le rapport minuscule / majuscule, l'autre le souligné, ... jusqu'à l'identité de ce tracé entre le « $A$ " du début avec le « $A$ » de la fin. La reproduction trouve alors son terme, son sens.

Dans la deuxième séquence, la différence opère de l'essence d'un acte signifiant. La différence ne porte pas sur la diversité, mais sur le seul fait qu'un caractère n'est pas celui adjacent. Un signifiant ne signifiant pas lui-même, il instaure la différence dans l'acte de répéter qui caractérise la chaîne signifiante. À se réitérer, le même en vient à écrire la différence. Le premier "a " n'est pas le second, ni le troisième, ...

Cette différence entre répétition et reproduction met en rapport l'insistance de la chaîne signifiante avec l'acte de ritualiser :

Tout ce qui, dans la répétition, se varie, se module, n'est qu'aliénation de son sens. L'adulte, voire l'enfant plus avancé, exigent dans leurs activités, dans le jeu, du nouveau. Mais ce glissement voile ce qui est le vrai secret du ludique, à savoir la diversité plus radicale que constitue la répétition en ellemême. Voyez-la chez l'enfant, dans son premier mouvement, au moment où 
il se forme comme être humain, se manifester comme exigence que le conte soit toujours le même, que sa réalisation racontée soit ritualisée, c'est-à-dire textuellement la même. (LACAN, 1963-64, p. 60; notre souligné)

Ritualiser met à jour l'écriture de la répétition, le tracement d'une différence dans ce qui apparaît textuellement le même récit. L'essence de cet ici et maintenant ${ }^{8}$ qui aura été n'est déjà plus tout à fait le même. Répéter dévoile la différenciation opérée par le signifiant.

Dire l'énoncé " $A$ est $A$ " " laisse ouverte la prétention que le premier " $A$ * est le même que le deuxième " $A$ ». Au contraire, Lacan relit cet énoncé avec la répétition signifiante en évoquant l'exemple du mot obsolète. Que dire de l'énoncé suivant : obsolète est obsolète? La deuxième occurrence se distingue de la première.

La différence surgit de l'expérience de la parole. Le langage humain structure un écart inconscient, une différence entre énonciation et énoncé, entre dit et non-dit, parole et effet de sens. À se répéter, même le même ne correspond plus textuellement ou littéralement au même. À tout le moins, il advient dans l'à-côté et n'en produit pas moins du sens.

Ritualiser écrit l'opération par laquelle du sens advient d'un système signifiant, soit la signifiance. En effet, aucun signifiant ne saurait se signifier luimême, même pas à l'ombre d'une convention entre le mot et la chose ${ }^{10}$. Il ne produit du sens que par concaténation avec d'autres signifiants, car « le signifiant en lui-même n'est rien d'autre de définissable qu'une différence avec un autre signifiant " (LACAN, 1972-73, p. 129). De là découle la primauté du signifiant sur le signifié exprimée partiellement par l'algorithme lacanien, signifiant sur signifié (LACAN, 1966, p.499). Cette primauté ne tient intrinsèquement qu'à la façon dont les signifiants peuvent signifier. Il ne s'agit que de la primauté exigée par la signifiance, et non pas d'une supériorité quelconque du signifiant sur le signifié. Reprenant l'exemple de la ritualisation du conte pour l'enfant (FREUD, 1976, p.45), Lacan y décèle cette primauté de la signifiance :

Cette exigence d'une consistance distincte des détails de son récit, signifie que la réalisation du signifiant ne pourra jamais être assez soigneuse dans sa mémorisation pour atteindre à désigner la primauté de la signifiance comme

8 Il s'agit de l'ipséité, à savoir ce qui fait qu'un être est lui-même et non un autre.

9 Les logiciens parlent d'énoncés tautologiques, $A \Leftrightarrow A, A$ équivalent à $A$.

10 La correspondance entre un mot et une chose établit un code faisant appel à des signes, et non pas à des signifiants. Pour Lacan, le signe représente quelque chose pour quelqu'un tandis qu'un signifiant représente le sujet pour un autre signifiant. Les signes communiquent de l'information mais ne laissent pas de place pour les versants métaphorique et métonymique qui caractérisent le langage humain. 
telle. C'est donc s'en évader, en apparence, que de la développer en variant les significations. Cette variation fait oublier la visée de la signifiance en transformant son acte en jeu,... (LACAN, 1963-64, p. 60; nous soulignons)

Ritualiser n'est pas un jeu imbu d'une pléthore d'éléments dont la diversité tiendrait lieu de nouveauté. Les rites singularisent l'unicité humaine en tant qu'ils sont de l'ordre de l'acte, c'est-à-dire de l'opération de la signifiance :

[...], c'est de partir de ceci, que la religion, parmi les modes que l'homme a de poser la question de son existence dans le monde, et, au-delà, la religion comme mode de subsistance du sujet qui s'interroge, se distingue par une dimension qui lui est propre, et qui est frappée d'un oubli. Dans toute religion qui mérite cette qualification, il y a en effet une dimension essentielle $\grave{a}$ réserver quelque chose d'opératoire, qui s'appelle un sacrement. (LACAN, 196364 , p. 239; nous soulignons)

Comment ne pas évoquer ici la présentation du rite selon Saint-Exupéry :

-Qu'est-ce qu'un rite? dit le petit prince.

- C'est aussi quelque chose de trop oublié, dit le renard. C'est ce qui fait qu'un jour est différent des autres jours, une heure, des autres heures. (Antoine de SAINT-EXUPÉRY, p. 70)

À ne pas se signifier lui-même, le signifiant opère de la différence à l'intérieur de la répétition. Remémoration de l'opération de la signifiance, le rite rappelle à l'humain sa particularité de sujet épris du signifiant : « Il y a, selon le discours analytique, un animal qui se trouve parlant, et pour qui, d'habiter le signifiant, il résulte qu'il en est sujet. " (LACAN, 1972-73, p.81).

\section{Synthèse de ce parcours et conséquence}

Freud situe les véritables mobiles aussi bien du cérémonial névrotique que des exercices religieux comme étant marqués d'un non-savoir. Ignorer ce nonsavoir contraint les rites à une entrée impossible, céder ou résister. Céder aux rites sans savoir leurs sens laisse place à l'illusion. Ils se trouvent pris dans une dynamique névrotique puisqu'ils opèrent à partir du sens inconnu ou ignoré. En revanche, donner sens à un rite au départ n'est pas moins problématique. Ce sens devrait mettre en lumière l'angoisse qui cause le recours au rite en luimême. Du coup, le rite perd sa pertinence. La mise à jour d'un sens qui cerne l'angoisse conduit à résister intrinsèquement au rôle masquant du rite.

La perspective freudienne ne réduit pas les rites au seul aboutissement névrotique. Freud ouvre une nouvelle perspective. Que le sens des rites soit symbolique ou non, il n'échappe pas à un lieu où le savoir est supposé. La problématique lacanienne poursuit et affine l'investigation freudienne à partir de ce 
point. Lacan décrit le lieu d'un tel rapport au savoir comme étant de l'ordre du transfert.

La place des rites trouve encore sa pertinence puisque des expressions ritualisées font partie de la relation de transfert. De plus, le transfert constitue la mise en scène du rapport entre l'inconscient et la répétition. La répétition tente de cerner le réel d'une rencontre immémorable. Unique pendant diurne du rêve, le rite constitue la voie royale de l'inconscient par sa façon d'écrire et de mettre en jeu la répétition.

La répétition dans le rêve opère de la chaîne signifiante; le rite relève de la même dynamique que le signifiant. Cet apport lacanien montre que le rite n'opère pas d'abord du symbole mais du signifiant. Comme le signifiant ne peut se signifier lui-même, chaque constituant d'un rite pris isolément se trouve aussi soustrait au sens. Pris isolément, le sens du rite semble en déroute. Ce point permet de rendre compte du surgissement de l'aporie du " céder ou résister ». Situer la dynamique des rites à partir d'un système signifiant ouvre le chemin pour trouver une pertinence. Ne chercher du sens que dans l'hétérogénéité des éléments manifeste parfois un refus de la différence. La soif d'une incessante diversité représente peut-être une résistance à toute forme de rite. De façon similaire, céder à la facile reproduction de l'identique sans jamais opérer la lecture de ce qui se répète convie à la platitude où s'engouffre la satisfaction du névrosé.

En conséquence de ce parcours, la réduction des rites à une impasse névrotique s'ouvre à une autre alternative tracée par Freud lui-même. La dynamique du " céder ou résister " trouve sa source dans l'opération même de la signifiance. Dès lors, elle peut se reformuler autrement à partir de l'acte de répéter par lequel un système signifiant opère. Ne pas céder à l'identique du signifiant, qui ne signifie rien et rien de moins que de ne pas être l'autre, c'est résister à l'acte d'écriture de la différence. La pratique de la parole n'est pas étrangère à ce dilemme.

Quant à la dimension symbolique, elle ne relève pas d'un sens particulier qui maintiendrait les rites hors du champ d'investigation des sciences humaines. Elle provient de l'inscription de l'humain dans le langage. Le sujet épris des signifiants se trouve divisé selon les effets métaphorique et métonymique du langage. Sans préjudice à leur importance, ces effets de sens surgissent de la relation entre les signifiants, peu importe le champ disciplinaire où ils se situent. Le rite est-il un jeu de diversion, ou une version du dit? $\AA$ tout le moins, il constitue une pratique discursive où le différent s'écrit à partir de la répétition du même. 


\section{Références bibliographiques}

FREUD, S.,

1980, L'interprétation des rêves. Paris, PUF.

1981, Malaise dans la civilisation. Paris, PUF.

1989, La technique psychanalytique. Paris, PUF.

HERVIEU-LÉGER, D.,

1994, "Religion, objet perdu et reconstruit : un parcours de recherche ", Religiologiques, no. 9, printemps, p. 31-41.

KREMER-MARIETTI, A.,

1978, Lacan et la rhétorique de l'inconscient. Paris, Aubier Montaigne.

LACAN, J.,

1953-54, Les écrits techniques de Freud. Livre I, Paris, Seuil, 1975.

1955-56, Les Psychoses. Livre III, Paris, Seuil, 1981.

1956-57, La relation d'objet. Livre IV, Paris, Seuil, 1994.

1959-60, L'éthique de la psychanalyse. Livre VII, Paris, Seuil, 1986.

1963-64, Les quatre concepts fondamentaux de la psychanalyse. Livre XI, Paris, Seuil, 1973.

1966, Écrits, Paris, Seuil.

1972-73, Encore, livre XX, Paris, Seuil, 1975.

1973-74, Les non-dupes errent, séminaire inédit.

LEMAIRE, A.,

1977, Jacques Lacan, Bruxelles, Pierre Mardaga.

SAINT-EXUPÉRY, A. de,

1982, Le Petit Prince. Paris, Gallimard.

ST-ARNAUD, G.-R.,

1996, "Aspect freudien des rites et de leurs répétitions ", Théologiques, vol. 4, no. 1, mars, p. 75-94.

SUBLON, R.,

1993, La lettre ou l'esprit. Une lecture psychanalytique de la théologie. Paris, Cerf. 